\title{
Basic Application of Deep Convolutional Neural Network to Visual Inspection
}

\author{
Fusaomi Nagata ${ }^{\mathrm{a},{ }^{*}}$, Kenta Tokuno ${ }^{\mathrm{a}}$, Hisami Tamano ${ }^{\mathrm{b}}$, Hitoshi Nakamura ${ }^{\mathrm{b}}$, Makoto Tamurab, \\ Kenjiro Kato ${ }^{\mathrm{b}}$, Akimasa Otsuka ${ }^{\mathrm{a}}$, Takeshi Ikeda ${ }^{\mathrm{a}}$, Keigo Watanabec, Maki K. Habib ${ }^{\mathrm{d}}$ \\ ${ }^{a}$ Department of Mechanical Engineering, Faculty of Engineering, Tokyo University of Science, Yamaguchi \\ Sanyo-Onoda 756-0884, Japan \\ ${ }^{\mathrm{b}}$ Gunma Research and Development Center, Mitsubishi Pencil Co. Ltd. \\ Fujioka, Gunma 375-8501, Japan \\ 'Graduate School of Natural Science and Technology, Okayama University \\ Okayama 700-8530, Japan \\ ${ }^{\mathrm{d}}$ Mechanical Engineering Department, School of Sciences and Engineering, The American University in Cairo \\ AUC Avenue, P.O.Box 74, New Cairo 11835, Egypt \\ *Corresponding Author: nagata@rs.tusy.ac.jp
}

\begin{abstract}
Artificial neural network (ANN) which has four or more layers structure is called deep $\mathrm{NN}$ (DNN) and is recognized as a promising machine learning technique. In this paper, a deep convolutional neural network (DCNN) is designed to inspect defects such as crack and burr phenomena occurred in the manufacturing process of resin molding, then the trained DCNN is evaluated through inspection experiments. A training image generator is first developed to systematically generate a lot of similar images for training. Similar images are efficiently produced by rotating, translating, scaling and transforming an original image. Then, the designed DCNN is trained using the images for the input layer and their categories for the output layer. The developed approach is assessed using the trained DCNN and shows the ability to classify sample images in a training test set into "OK" or "NG" category with high accuracy.
\end{abstract}

Keywords: Deep learning, Deep convolutional neural network, Visual inspection system, Training image generator.

\section{Introduction}

Artificial neural network (ANN) which has four or more layers structure is called deep NN (DNN) and is recognized as a promising machine learning technique. Nagi et al. designed max-pooling convolutional neural networks (MPCNN) for vision-based hand gesture recognition ${ }^{(1)}$. The MPCNN could classify six kinds of gestures with 96\% accuracy and allow mobile robots to perform real-time gesture recognition. Weimer et al. also designed a deep CNN architectures for automated feature extraction in industrial inspection process ${ }^{(2)}$. The convolutional neural network (CNN) automatically generates features from massive amount of training image data and demonstrates excellent defect detection results with low false alarm rates. FaghihRoohi et al. presented a different type of deep CNN for automatic detection of rail surface defects ${ }^{(3)}$. It was concluded that the large CNN model performed a better classification result than the small and medium CNN, although the training required a longer time. Further, Zhou et al. used a CNN to classify the surface defects of steel sheets ${ }^{(4)}$. The CNN could directly learn better representative features from labeled images of surface defects.

In this paper, a DCNN is designed to inspect (detect) defects such as crack and burr phenomena occurred in the manufacturing process of resin molding, then the trained DCNN is evaluated through inspection trials. A training image generator is first developed to efficiently generate a lot of similar images for training by rotating, translating, scaling or transforming an original image. Then, the designed DCNN is trained using the images. The ability of the trained DCNN for classifying sample images in a training test set into "OK" or "NG" category is tested and evaluated.

\section{Preparation of Training Data}

Figures 1 and 2 show examples of "OK" and "NG" 
samples for training. Numerous image files of produced workpieces and their paired labels for classification are needed to construct a reliable DCNN system. To cope with the need, a dialogue application called the training image generator was first developed for DCNN training that can efficiently produce a lot of similar images from an original one as shown in Fig. 3, in which similar deformed images are generated by rotating, translating, scaling or transforming an original image. For example, the images given with the number from 89 to 100 in Fig. 1 are similar but a little bit different. They are efficiently increased by rotating, translating or scaling the original image.

A training set consisting of 2040 samples, i.e., 1020 "OK" and 1020 "NG" samples, is prepared in advance. "NG" samples include multiple images with undesirable crack, burr and/or knob (protrusion). It is expected that the DCNN designed in the next section is able to obtain the promising cognition ability to categorize samples into "OK" or "NG".

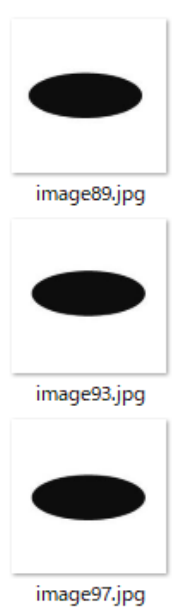

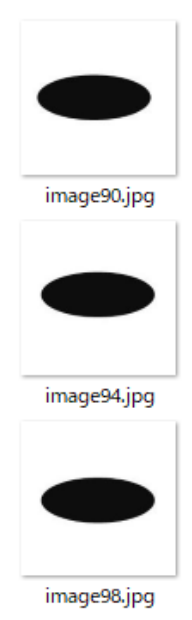

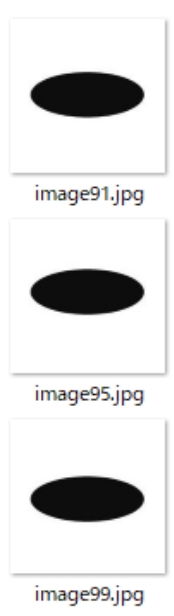

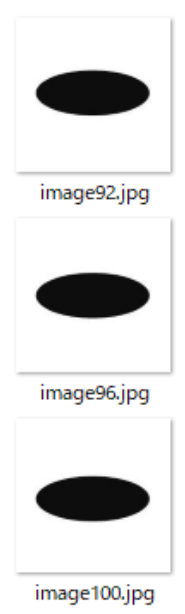

Fig. 1. Image samples of "OK” products.

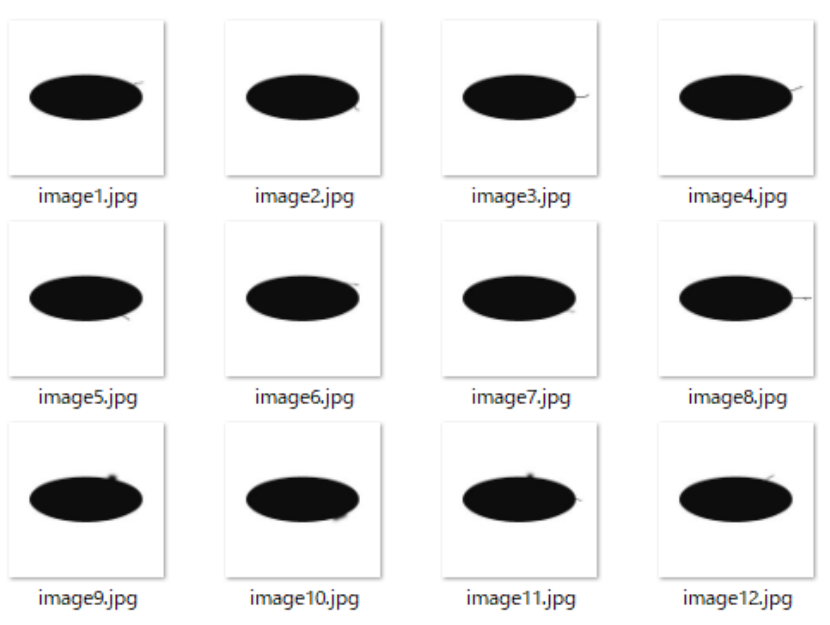

Fig. 2. Image samples of "NG" products, in which crack, burr and/or protrusion are seen.

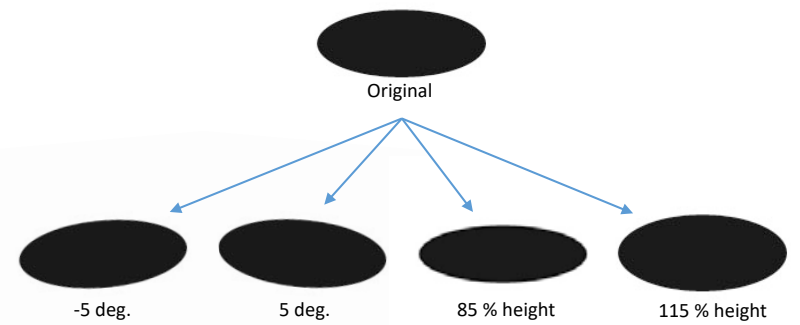

Fig. 3. Transformed images from an original one.

\section{Design of Deep Convolutional Neural Network}

Figure 4 illustrates the designed DCNN with 15 layers. The structure of the DCNN is listed as follows:
1. Image Input
2. Convolution
3. Rectified Linear Unit (ReLU)
4. Max Pooling
5. Convolution
6. ReLU
7. Max Pooling
8. Convolution
9. ReLU
10. Max Pooling
11. Fully Connected
12. ReLU
13. Fully Connected
14. Softmax
15. Classification Output

The first input layer is placed for input images $(200 \times 200$ $\times 1$ ) with 1 channel 8 bits gray scale given by a matrix with zerocenter normalization. The $2^{\text {nd }}$ and $5^{\text {th }}$ layers are convolution ones which have 32 filters with the resolution of $5 \times 5 \times 1$. In the convolution layers, the filters are applied to a training image one by one while being shifted from the left top to the right bottom. The $8^{\text {th }}$ layer is convolution one which has 64 filters with the resolution of $5 \times 5 \times 1$. Each pixel in the filters has also a value of 8 bits gray scale. The sizes of stride and padding are set to 1 and 2 in training, respectively. The stride and padding are important parameters. The amount by which a filter shifts is called the stride. The stride controls the degree of convolution in a training image. The layers 3, 6, 9, 12 are activation functions 


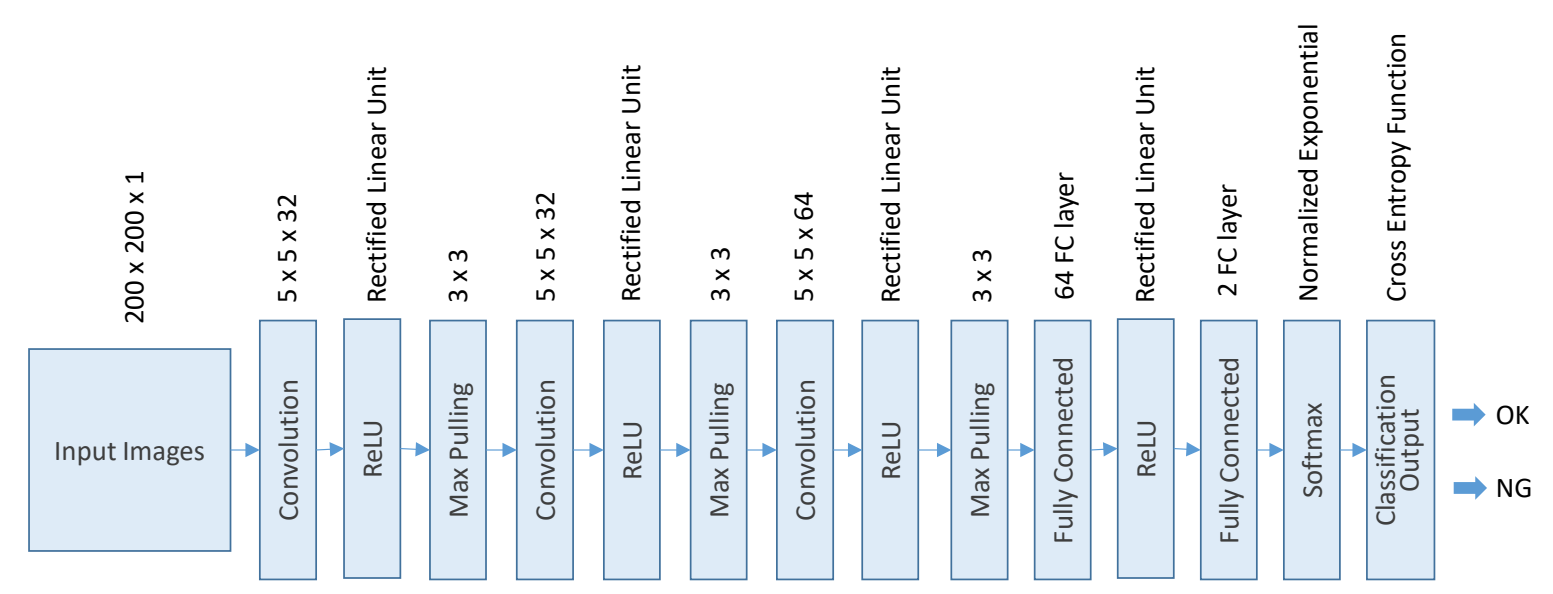

Fig. 4. Designed deep neural network consisting of convolution, ReLU, pooling, softmax and classification layers.

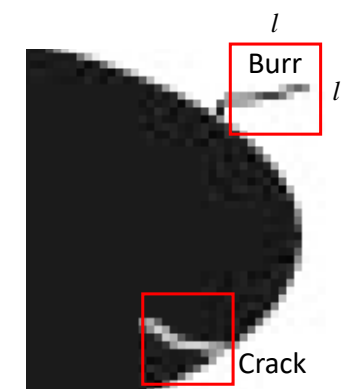

Fig. 5. Filter size for efficiently obtaining feature maps.

called Rrectified Linear Unit (ReLU) given by $f(u)=\max (0$, $u)$. The $14^{\text {th }}$ softmax layer calculates the probabilities $p_{i}(i=$ $1,2)$ of two categories "OK" and "NG" by

$$
p_{i}=\frac{\exp \left(y_{i}\right)}{\exp \left(y_{1}\right)+\exp \left(y_{2}\right)}
$$

where $y_{1}$ and $y_{2}$ are the outputs from the $13^{\text {th }}$ fully connected layer.

Figure 5 shows the image of square filter to detect typical defects such as burr, crack, and so on.

\section{Training Experiment}

Table 1 tabulates the main parameters for training of the DCNN. The training was conducted by using a single PC with a Core i7 CPU and a GPU (NVIDIA GeForce GTX 1060, 6GB). In this DCNN design, it took about several minutes until the training accuracy reached to 0.95 .

After the DCNN was trained, a categorization test was conducted using the training set. Figure 6 shows the scores of categorization "OK" and "NG" using all the samples in training set (total samples number is 2040). As can be seen, all the 2040 samples can be well identified with the accuracy more than 0.97 .

The generalization of the trained DCNN was simply evaluated using the training test samples shown in Fig. 7 which were not included in the training set. In other words, the training test set has not been ever used for training. Figure 8 shows the result of the accuracies of categorization "NG" using the 10 training test samples, in which it is observed that “image2.jpg” and "image9.jpg” are not well categorized. For example, Fig. 9 shows the enlarged figure of the defect area of "image2. jpg" in Fig. 8.

Figure 10 shows the additional 10 training samples which are a little bit deformed from "image2.jpg" in Fig. 8. To enhance the categorization ability further to the samples given by Figs. 7 and 10, the trained DCNN was retrained using the reorganized training set consisting of original 2040 samples, 10 "NG" ones in Fig. 7, 10 "NG" ones in Fig. 10, and additional 20 "OK" ones. Figure 11 shows the scores of categorization "OK" and "NG" using all the samples in reorganized training sets (total samples number $=1040+$ 1040) including the samples in Figs. 7 and 10. It is observed from Fig. 11 that the recognition ability to additional "NG" samples can be improved effectively and pinpointedly.

Table 1. Parameters of DCNN training.

\begin{tabular}{l|c}
\hline Filter size & 5 \\
Padding & 2 \\
Stride & 2 \\
Max epochs & 30 \\
Mini batch size & 200 \\
Learning Rate & 0.002 \\
Desired training accuracy & 0.95 \\
Sample number of "OK" & 1020 \\
Sample number of "NG" & 1020 \\
\hline
\end{tabular}




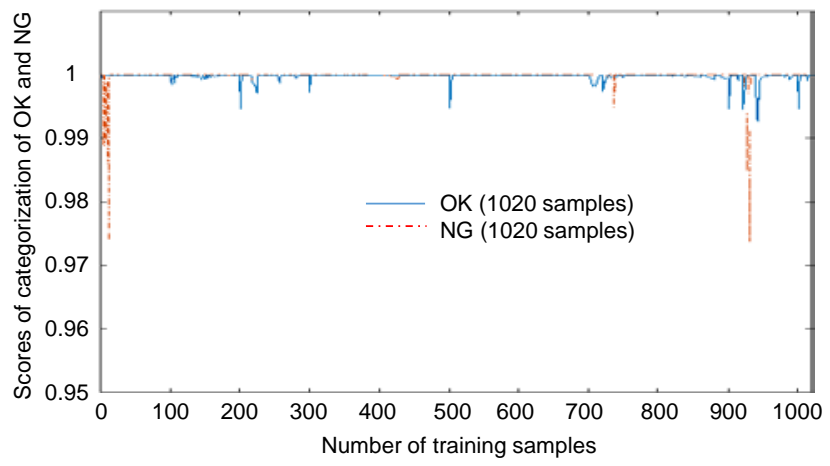

Fig. 6. Scores of categorization "OK” and "NG" using the samples in training set (samples number $=1020+1020$ ).

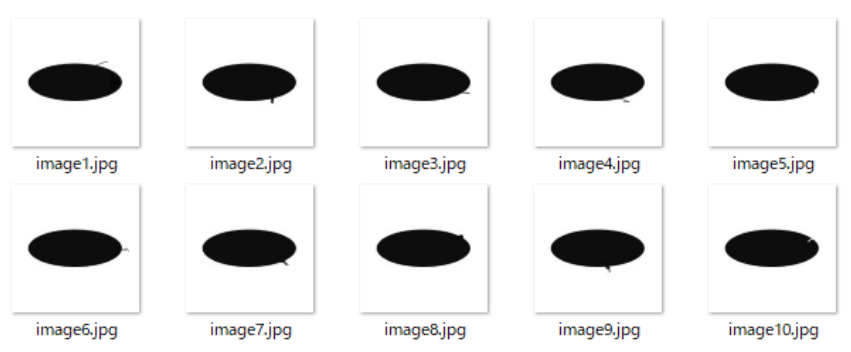

Fig. 7. Training test samples of categorization "NG", which were not included in the training set.

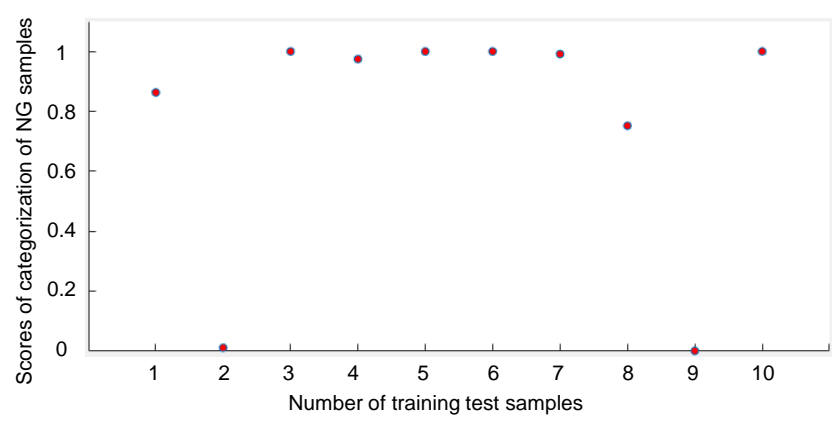

Fig. 8. Scores of categorization "NG" using 10 training test samples which mean unlearned samples.

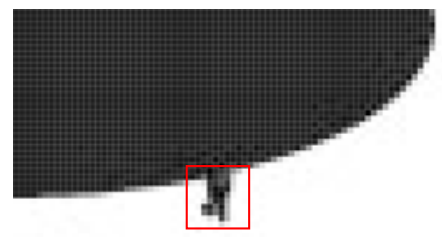

Fig. 9. Enlarged figure of a defect of “image2.jpg” in Fig. 8.

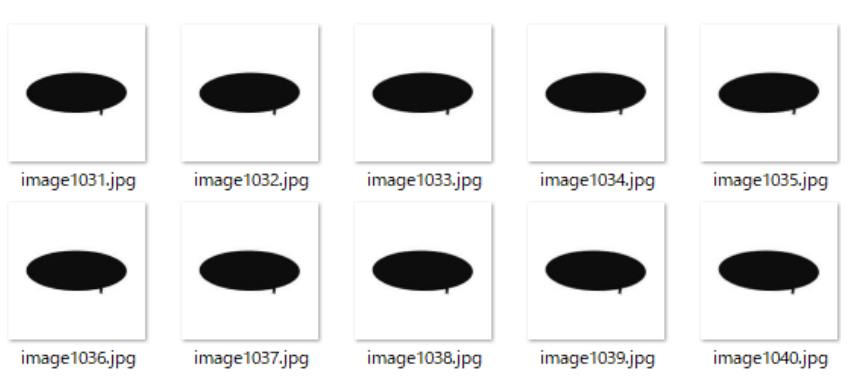

Fig. 10. Additional 10 training samples a little bit deformed from “image2.jpg” in Fig. 8.

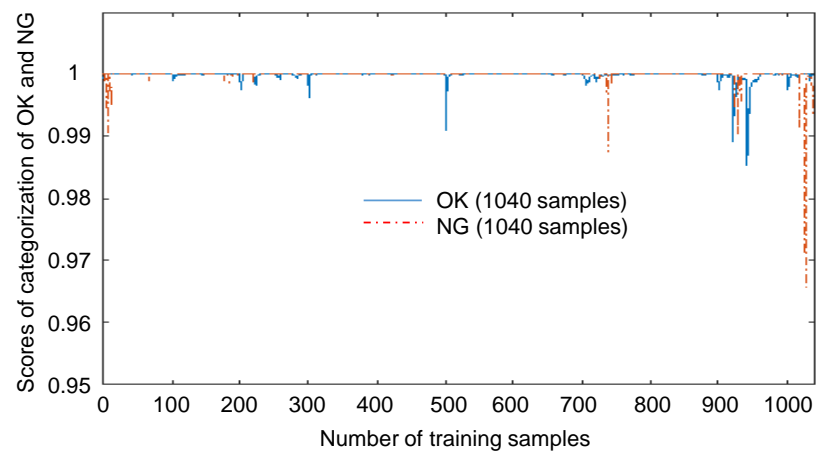

Fig. 11. Scores of categorization "OK" and "NG" using the samples in training sets (total samples number $=1040+1040$ ), in which samples shown in Figs. 7 and 10 are added.

\section{Conclusions}

Artificial neural network (ANN) which has four and more layers structure is called deep $\mathrm{NN}$ (DNN) and is recognized as a promising machine learning technique. In this paper, a DCNN was designed to inspect (detect) defects such as crack and burr phenomena occurred in the manufacturing process of resin molding, then the trained DCNN was evaluated. A training image generator was first developed to efficiently generate a lot of similar images for training from an original one. The DCNN was efficiently trained using the generated images. It has been suggested that the trained DCNN has a promising ability to be able to classify sample images in training test set into "OK" or "NG". Furthermore, the additional training method to cope with not well trained samples was shown and evaluated, so that the recognition ability could be effectively and pinpointedly improved to a level of categorization score over 0.96 . The designed DCNN is expected to be able to be applied to an actual inspection process. 


\section{Acknowledgment}

This work was supported by JSPS KAKENHI Grant Number JP16K06203.

\section{References}

(1) J. Nagi, F. Ducatelle, G.A.D. Caro, D. Ciresan, U. Meier, A. Giusti, F. Nagi, J. Schmidhuber, and L.M. Gambardella : "Max-pooling convolutional neural networks for vision-based hand gesture recognition," 2011 IEEE International Conference on Signal and Image Processing Applications (ICSIPA2011), pp. 342-347, 2011

(2) D. Weimer, B. Scholz-Reiter, and M. Shpitalni : "Design of deep convolutional neural network architectures for automated feature extraction in industrial inspection,” CIRP Annals - Manufacturing Technology, Vol. 65, No. 1, pp. 417-420, 2016

(3) S. Faghih-Roohi, S. Hajizadeh, A. Nunez, R. Babuska, and B.D. Schutter : "Deep convolutional neural networks for detection of rail surface defects,” Procs. of the 2016 International Joint Conference on Neural Networks (IJCNN 2016), Vancouver, Canada, pp. 2584-2589, July 2016

(4) S. Zhou, Y. Chen, D. Zhang, J. Xie, and Y. Zhou : "Classification of surface defects on steel sheet using convolutional neural networks,” Materials and Technology, Vol. 51, No. 1, pp. 123-131, 2017 